\title{
On the Persistence of Dominant Inter-Area Oscillation Paths in Large-Scale Power Networks
}

\author{
Yuwa Chompoobutrgool* Luigi Vanfretti* \\ * KTH Royal Institute of Technology, Stockholm, Sweden. \\ (E-mail: yuwa@ee.kth.se, luigiv@kth.se)
}

\begin{abstract}
This article discusses the degree of persistence of dominant inter-area oscillation paths: the passageways containing the highest content of inter-area oscillations in large-scale power networks. To this aim, a number of contingency studies are carried out: faults being imposed on the lines connecting to the dominant inter-area path. The path persistence is then examined using the relationship between sensitivities of network variables (i.e. voltages and current, magnitude and angle), and mode shapes describing the contribution of the oscillations among the generators variables (e.g. speed and angle). This relationship is termed network modeshape. The larger in magnitude and the lesser the variation the network modeshape is, the more observable and the more robust the signals measured from the dominant path becomes. The outcome is a proposed signal combination to be used as inputs to the damping controller for mitigation of inter-area oscillations in large-scale power systems.
\end{abstract}

Keywords: Inter-area oscillations; power systems; large-scale power networks; sensitivity analysis; eigenvalue computations; network modeshapes; dominant path.

\section{INTRODUCTION}

Electric power systems are one of the most complex engineering networks of today's critical infrastructure. They are comprised of a large number of blocks, where each consists of numerous sub-systems within the system as a whole (e.g. a synchronous generator with controls), making power networks challenging to analyze and understand. With the continuous increase in electricity demand and the trend for more interconnections [Sattinger (2011)], one issue of concern is the mitigation of low-frequency inter-area oscillations (LFIO). Typically, inter-area oscillations occur in large power systems interconnected by weak transmission lines [Chow (1982)] that transfer heavy power flows. Usually, these oscillations have the critical characteristic of poor damping. When a certain type of swing occurs in such system, insufficient damping LFIOs may lead to a limitation of power transfer capability or, worse than that, a growth in amplitude of the LFIOs which could possibly cause a system to collapse [Rogers (1996)].

To enhance transfer capacity while preventing the system from breaking up, a common countermeasure is to install power system stabilizers (PSS), which provide additional damping to the system at generators. Successful damping, however, relies heavily on the locations and types of input signals to PSS, as well as the PSS locations. Input signals can be categorized into two groups: local and "global" (also known as wide-area [Glavic and Van Cutsem (2009)]) signals. Although it has been shown in several studies that the former are less preferable to the latter [Kamwa et al. (2006); Chow et al. (2000); Kamwa et al. (2005)], at present, global signals are seldom used in practice. In fact, two of the major challenges in the field are to adequately

^ Y. Chompoobutrgool is supported by Elforsk, Sweden.

Luigi Vanfretti is supported by the STandUP for Energy collaboration initiative and the KTH School of Electrical Engineering. utilize global signals, and to select "appropriate" input signal types for power oscillation damping control; i.e., signals with high robustness and observability.

With the advent of phasor measurement units (PMU), not only global signals but also other types of system variables, e.g. voltages or currents, are becoming available [Phadke and Thorp (2008)]. In contrast to conventional input signals such as generator speed or electrical power, which are not easily obtained globally, PMUs offer timely synchronized direct measurement of network variables. It is, therefore, our aim to find the set of signals readily available by PMUs, using an effective and systematic method, to be used as input signals to PSSs and other damping controllers. It is expected that, using the globally available and properly selected signals obtained from PMUs, effective damping of LFIOs will be achieved. Consequently, more power could be transferred while the system maintains its required degree of security.

\section{ASSUMPTION AND HYPOTHESES}

The concept of "interaction paths" as the group of transmission lines, buses and controllers which the generators in a system use for exchanging energy during swings has been useful for characterizing the dynamic behaviour of the Western Electric Coordinating Council (WECC) power system. In [Hauer et al. (2007)], interaction paths in the WECC have been determined by performing active power oscillation signal correlation from one important line against all other key lines in the network. This analysis showed that the interaction between two distantly located transmission lines was apparent from a coherency function, thus allowing to locate transmission corridors with relevant oscillatory content in the measured signals passing through them. The long experience of the WECC in the determination of this complex network's most important paths has been carried out through a signal analysis approach 
using multiple measurement archives; this is a vigorous chore for such a complex and large interconnected network. For predominantly radial systems, fortunately, it is more straightforward to determine interaction paths. As an example, consider the power system in Queensland [Ledwich and Palmer (2000)] where the main oscillation modes interact through radial links, one from the north to the center of the system, and a second from the south to the center of the system. Here the interaction paths are obvious and pre-determined by the radial nature of the transmission network and allocation of generation sources.

This article builds on these observations and attempts to bridge the gap in the understanding of the so-called "interaction paths" and their behavior. From the discussion above it is assumed that the propagation of interarea oscillations in inter-connected system is deterministic [Chow (1982)]; i.e., the oscillation always travels in certain paths, and the main path can be determined a priori ${ }^{1}$. This path is denominated as the dominant inter-area path: the passageway containing the highest content of the interarea oscillations. The deduction of dominant paths is not within the scope of this article, but will be described in another publication.

With this assumption, two hypotheses are made in this study: (1) network signals from the dominant path are the most visible among other signals within a system, and they have the highest content of inter-area modes (these signals may be used for damping control through PSS or other damping controllers), and (2) there is a degree of persistence to the existence of the dominant path; i.e. it will be consistent under a number of different operating conditions and the signals drawn from it will still be robust and observable.

The objective of this paper is, then, to show the degree of persistence of the dominant inter-area path. To this aim, a number of contingency studies are carried out to corroborate the hypotheses. Once persistence is verified, a set of qualified input signals from the path will be investigated and their use for damping of inter-area oscillations will be theorized.

The remainder of this article is organized as follows. In Section 3, the contingency studies, some important concepts used in the study, methodology, and the characteristics of the test system are described. Results from contingency studies and the corresponding discussion are provided in Section 4. Using the results from the previous sections, a set of feedback signals are proposed and verified by means of a small disturbance in Section 5. Conclusions are finally presented in Section 6.

\section{CONTINGENCY STUDIES AND ANALYSIS METHODOLOGY}

\subsection{Contingency Studies}

Contingencies considered in this study are loss of transmission lines, including those directly connecting to the dominant inter-area path, and are denoted by far, near, and on. The classification of each event is determined by how "close" the line to be removed is to the pre-determined dominant path. The path persistence is then examined from the relationship between two key factors: sensitivities

1 Give that the network structure is not drastically modified. of network variables and mode shapes. This relationship is termed network modeshape [Vanfretti and Chow (2010)]. Their definitions are described next.

\subsection{Important Concepts}

Mode Shape-denoted by $W(A)$, is an element describing the distribution of oscillations among system's state variables. In mathematical terms, it is the right eigenvector obtained from an eigenanalysis of a linearized system. The mode shapes of interest here are those that belong to electromechanical oscillations, of which the corresponding state variables are generator rotor angles $(\delta)$ and speed $(\omega)$. Mode shape plots give directions of the oscillations and, thus, are used to determine groups of generators. The derivations of electromechanical mode shapes will be briefly described here. Consider a linearized $N$-machine system in a state-space form

$$
\Delta \dot{x}=A \Delta x+B \Delta u, \quad \Delta y=C \Delta x+D \Delta u,
$$

where vectors $\Delta x, \Delta y$, and $\Delta u$ represent the state variables, the output variables and the inputs, respectively. With no input, the electromechanical model is expressed as

$$
\underbrace{\left[\begin{array}{c}
\Delta \dot{\delta} \\
\Delta \dot{\omega}
\end{array}\right]}_{\Delta \dot{x}}=\underbrace{\left[\begin{array}{ll}
A_{11} & A_{12} \\
A_{21} & A_{22}
\end{array}\right]}_{A} \underbrace{\left[\begin{array}{c}
\Delta \delta \\
\Delta \omega
\end{array}\right]}_{\Delta x}
$$

where matrix $A$ represents the state matrix corresponding to the state variables $\Delta \delta$ and $\Delta \omega$. Then, performing eigenanalysis, the electromechanical mode shape is derived from $A W(A)=\lambda W(A)$ where $\lambda$ are eigenvalues of the electromechanical modes of the system. Inter-area oscillations, as well as other modes, are determined from the eigenvalues.

Network Sensitivities - The sensitivities of interest are those from network variables; namely, bus voltage phasors with respect to change in the state variables, e.g. machine's rotor angle or speed. Since PMUs provide measurement in phasor form, the analyses in this study regard two quantities: voltage magnitude $(V)$ and voltage angle $(\theta)$. That is, the network sensitivities are the $C$ matrix from (1) with voltage magnitude and angle as the outputs $\Delta y$. Sensitivities of the voltage magnitude $\left(C_{V}\right)$ and voltage angle $\left(C_{\theta}\right)$ are expressed as

$\underbrace{\left[\begin{array}{c}\Delta V \\ \Delta \theta\end{array}\right]}_{\Delta y}=\underbrace{\left[\begin{array}{ll}\frac{\partial \Delta V}{\partial \Delta \delta} & \frac{\partial \Delta V}{\partial \Delta \omega} \\ \frac{\partial \Delta \theta}{\partial \Delta \delta} & \frac{\partial \Delta \theta}{\partial \Delta \omega}\end{array}\right]}_{C} \underbrace{\left[\begin{array}{c}\Delta \delta \\ \Delta \omega\end{array}\right]}_{\Delta x}=\left[\begin{array}{ll}C_{V} & C_{\theta}\end{array}\right]^{T}\left[\begin{array}{c}\Delta \delta \\ \Delta \omega\end{array}\right]$

Network Modeshapes - as introduced in [Vanfretti and Chow (2010); Vanfretti (2009)], network modeshapes $(S)$ are the projection of the network sensitivities onto the electromechanical modeshape, which is computed from the product of network sensitivities and mode shapes. It indicates how much the content of each mode ${ }^{2}$ is distributed within the network variables. In other words, how "observable" the voltage signals on the dominant path are for each mode of oscillation. The expressions for voltage magnitude and voltage angle modeshapes $\left(S_{V}\right.$ and $\left.S_{\theta}\right)$ are

$$
S_{V}=C_{V} W(A), \quad S_{\theta}=C_{\theta} W(A) .
$$

2 This article focuses on inter-area modes, however, the theory is general and can be applied to any mode. 
It can therefore be realized that the larger in magnitude and the lesser in variation the network modeshape is (under different operating points), the more observable and the more robust the signals measured from the dominant path become.

\subsection{Methodology}

A step-by-step procedure performed in this contingency study is described as follows.

(1) Perform a power flow of the nominal, i.e. unperturbed, system to obtain initial conditions of all network variables.

(2) Perform linearization to obtain the network sensitivities $\left(C_{V}\right.$ and $\left.C_{\theta}\right)$.

(3) Perform eigenanalysis to obtain mode shapes $(W(A))$ and identify the inter-area modes and the corresponding dominant path. ${ }^{3}$

(4) Compute network modeshapes $\left(S_{V}, S_{\theta}\right)$.

(5) Plot $S_{V}$ and $S_{\theta}$ of the dominant inter-area path.

(6) Implement a contingency by removing a line.

(7) Repeat 1-5 (excluding the dominant path identification) and compare the results with that of the original case.

(8) Reconnect the faulted line and go to 6) for subsequent contingencies.

3.4 Test System and its Dominant Inter-Area Oscillation Paths

The system under study, namely KTH-NORDIC32 (see Fig. 1), is a conceptualization of the Swedish power system and its neighbors circa 1995; it was constructed from the power system data proposed by T. Van Cutsem [Van Cutsem (2010)] and further modified in [Chompoobutrgool and Vanfretti (2011); Chompoobutrgool et al. (2012)]. It has 20 generators, 12 of which are hydro generators located in the North and Equivalent areas, whereas the rest are thermal generators located in the Central and South areas. The northern regions (the North and Equivalent) account for most of the generation causing a heavy power transfer to the southern regions (the Central and South) through weak transmission lines. As a result, this looselyconnected system exhibits lightly damped LFIOs. The mode of concern in this study is the $0.49 \mathrm{~Hz}$ mode, with the lowest damping ratio, which denotes the swing between two groups of generators: the northern regions against those of the southern regions. In addition, the system has a second inter-area mode that is not discussed in this paper, see [Chompoobutrgool and Vanfretti (2011)] for details. Dominant inter-area oscillation paths are defined as the corridors within a system with the highest content of the inter-area oscillations, they have the following important features:

- The largest $S_{V}$ or the smallest $S_{\theta}$ element(s) indicates the center of the path. This center can be theorized as the "inter-area mode center of inertia" or the "inter-area pivot" for each of the system's inter-area modes.

- The difference between $S_{\theta}$ elements of two edges of the path are the largest among any other pair within the same path. In other words, the oscillations are the most positive at one end while being the most negative at the other end. Hence, they can be theorized as the "tails" for each inter-area mode path.

3 The deduction of dominant paths will be presented in another publication. Here, the dominant path is known a priori.

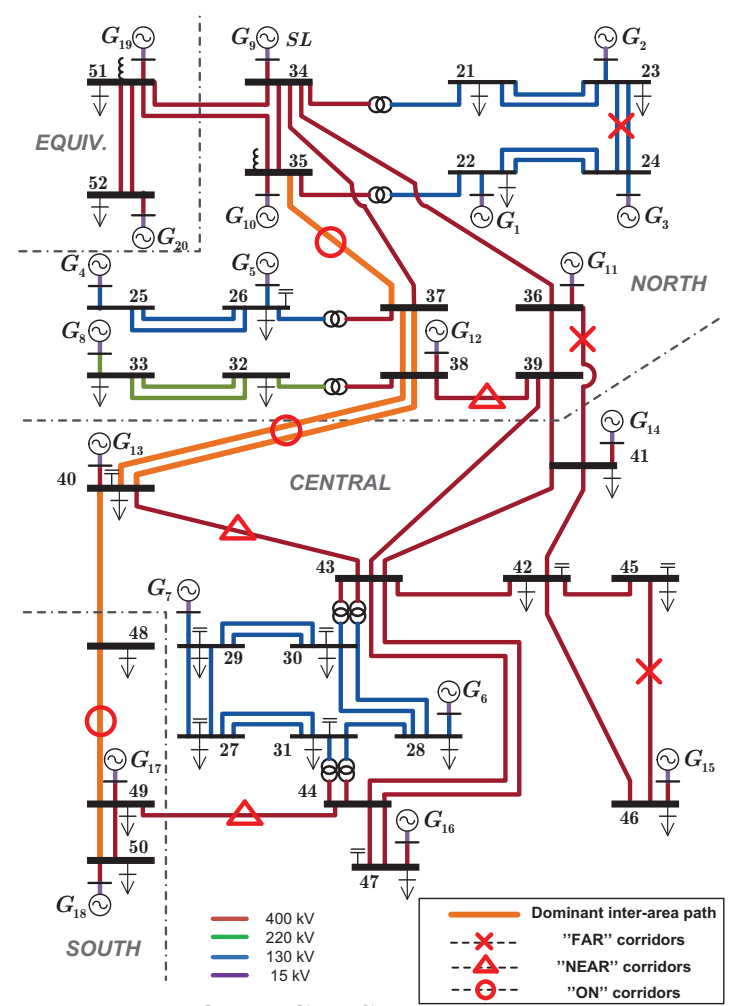

Fig. 1. KTH-NORDIC32 System.
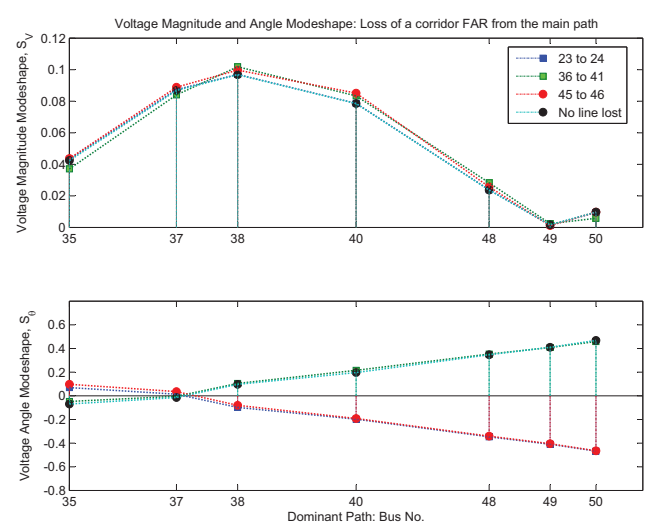

Fig. 2. Voltage magnitude and angle modeshapes: Loss of a corridor FAR from the dominant path.

- $S_{V}$ elements of the edges are the smallest or one of the smallest within the path.

The KTH-NORDIC32 system's dominant inter-area path is the corridors 35-37-37-40-48-49-50, which is highlighted in orange (see Fig. 1). To develop a fundamental understanding, the detailed model in Chompoobutrgool et al. (2012) is stripped from controllers and has no damping.

\section{SIMULATION RESULTS AND DISCUSSIONS}

Due to space restrictions, only selected simulation results will be shown in this paper. In Figs. 2 - 4, the $y$-axis of the upper and lower figures display the voltage magnitude and voltage angle modeshapes of the dominant inter-area oscillation path for each contingency, respectively. The $x$ axis represents the bus number in the dominant path; the distance between buses are proportional to the line impedance magnitude. For every scenario, the removal of corridors are compared to the nominal system denoted by black dots to determine the path's persistence. 

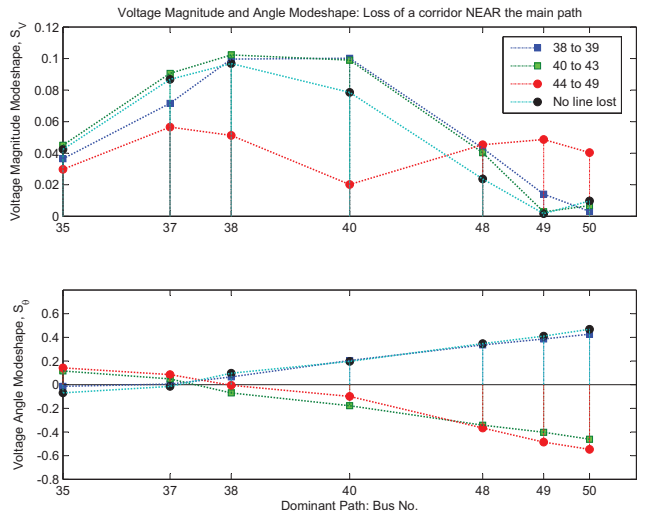

Fig. 3. Voltage magnitude and angle modeshapes: Loss of a corridor NEAR the dominant path.
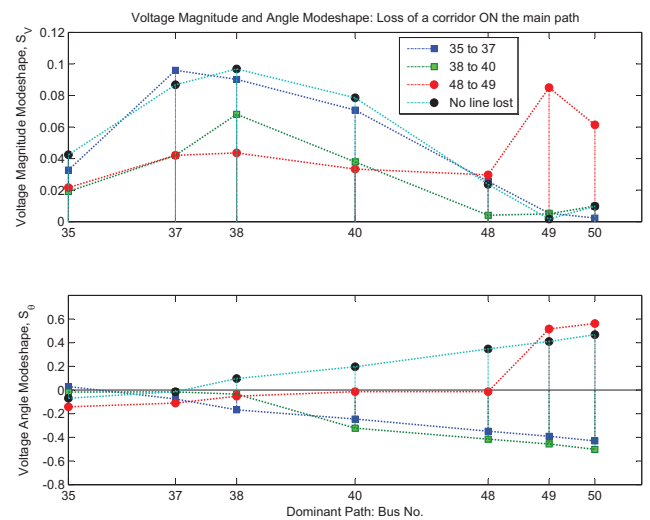

Fig. 4. Voltage magnitude and angle modeshapes: Loss of a corridor ON the dominant path.

\subsection{Loss of a corridor FAR from the dominant path}

Figure 2 shows the three selected corridors: 23-24, 3641, and 45-46, which are located the farthest from the dominant path as indicated by red "X" marks in Fig. 1. The results show that the voltage magnitude modeshapes $\left(S_{V}\right)$ remains consistent both in magnitude and direction, although there are small but insignificant variations. However, despite maintaining nearly the same magnitude as that of the nominal case, the voltage angle oscillations $\left(S_{\theta}\right)$ have opposite directions when the corridors 23-24 and 4546 are disconnected. Similar results are obtained with the removals of some other "FAR" corridors.

\subsection{Loss of a corridor NEAR the dominant path}

Figure 3 shows the three selected corridors: 38-39, 40-43, and 44-49, which are directly connected to the dominant path as indicated by red " $\Delta$ " marks in Fig. 1 . It can be observed that the removal of corridor 44-49 results in a significant reduction in the voltage magnitude modeshape, particularly, that of Bus 40. This is due to the following reasons: (1) Bus 49 is connected close to $G_{18}$ (Generator No.18) in which its speed variable is the most associated state in the $0.49-\mathrm{Hz}$ inter-area mode, and (2) Bus 40 is directly connected with $G_{13}$ which is a synchronous condenser.

The removal of the other corridors "NEAR" the main path has similar results to that of the removal of corridor 38-39; only small variations in both $S_{V}$ and $S_{\theta}$. The change in direction of $S_{\theta}$ (given by a sign inversion) only occurs with the disconnection of corridors 40-43 and 44-49.
Figure 4 shows the three selected corridors: 35-37, 38-40, and $48-49$, which belong to the dominant path as indicated by red "O" marks in Fig. 1. The removal of corridor 35-37 has a trivial effect, in terms of magnitudes, on both $S_{V}$ and $S_{\theta}$. On the contrary, the removal of corridors 38-40 or 48-49 has detrimental effects on $S_{V}$ and/or $S_{\theta}$. Particularly, that of the latter, the $S_{\theta}$ elements are close to zero in most of the dominant transfer path buses (except Bus 49 and Bus 50 ), although $S_{V}$ elements are still visible. In addition, although not shown here, the removal of corridor 37-38 results in non-convergent power flow solution while the removal of corridor 49-50 results in the disappearance of the "known" inter-area mode.

\subsection{Discussions}

The contingency studies above allow to recognize the following attributes of dominant paths:

- In most of the contingencies, the dominant path is persistent; the network modeshapes of voltage magnitude and angles maintain their visibility and strength (amplitude) as compared with the nominal scenario.

- In nearly all of the contingencies, despite small variations in the voltage magnitude modeshapes, the voltage angle modeshapes maintain their strength. However, the signs are in opposite direction in some of the cases. This sign change can be explained by a reversal in the direction of their corresponding mode shapes.

- In some contingencies such as the removal of corridor 49-50, the system topology is severely changed and the mode of interest disappears. The dominant path loses its persistence, and, due to the topological change, it ceases to exist giving rise to a different dominant path with different mode properties (frequency and damping). This indicates that $G_{18}$ is the origin of the $0.49 \mathrm{~Hz}$ mode. Thus, it can be inferred that corridor 49-50 is one of the most critical corridors for this inter-area mode distribution.

\section{CONSTRUCTING CONTROL INPUT SIGNALS}

Based on the results in the previous section, suitable network variables from the dominant path to construct PSSs input feedback signals are proposed here. Block diagram representations of how the signals could be implemented in practice are illustrated in Fig. 5. Latencies, e.g. communication and process delays, are omnipresent and play a critical role in damping control design. Nevertheless, in order to build a fundamental understanding they are neglected in this study, but will be considered in a future article.

To justify signal selection, a small disturbance is applied at linearized test system and the time responses of the selected outputs are simulated and analyzed. The perturbation is a variation of 0.01 p.u. in mechanical power

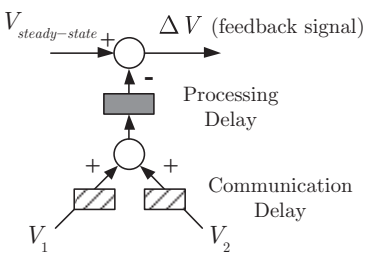

(a) Voltage magnitudes.

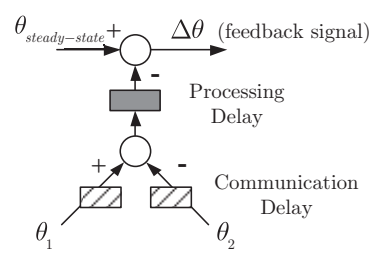

(b) Voltage angles.
Fig. 5. Block diagrams for the feedback signals. 
$\left(\Delta P_{M}\right)$ at selected generators and applied at $t=1 \mathrm{~s}$, and the system response is simulated for a period of $20 \mathrm{~s}$. The signals considered here are:

- Voltage magnitude deviation: $V_{23}, V_{37}, V_{38}, V_{48}, V_{50}$, and $V_{37}+V_{48}$

- Voltage angle difference: $\Delta \theta_{37,23}, \Delta \theta_{37,38}, \Delta \theta_{37,48}$, and $\Delta \theta_{37,50}$.

Bus 23 represents a non-dominant-path bus whereas the rest belong to the main path. The deviation in the voltage magnitude is the difference between the steady state and the simulated response, while that of the voltage angle is the variation among the simulated output signals, bus voltage angle $\theta_{37}$ is used as a reference. The set $V_{37}+V_{48}$ is used as as example of a signal combination of voltage magnitudes, while all the angle differences are inherently signal combinations.

To implement the network modeshape concept, the multimodal decomposition framework [Wei (2002)] is employed. It is an approach used to assess a complex multi-machine system with multiple swing modes. Mode shapes of the synchronizing coefficient matrix $A_{21}$ (see (2)) are incorporated with the linearized state-space model, thereby reconstructing the system. In this study, a partial multimodal decomposition [Larsen et al. (1995)] concept is used to evaluate one mode at a time, namely, the interarea oscillation modes. With this approach, the network modeshape, a product of mode shapes and sensitivities, is used as a filter allowing only the mode of interest to be evaluated.

\section{Partial Multi-Modal Decomposition}

Partial multi-modal decomposition computes from (2), $M$, the mode shapes of the matrix $A_{21}$, and the relationship $A_{21} \lambda_{21}=\lambda_{21} M, M^{-1} A_{21} M=\Lambda$ where $\Lambda$ is a matrix containing normalized modal synchronizing coefficients in the diagonal. Then, the system (1) is transformed into

$$
\Delta \dot{x}_{m}=A_{m} \Delta x_{m}+B_{m} \Delta u, \quad \Delta y=C_{m} \Delta x_{m}+D \Delta u
$$

where

$$
\begin{array}{r}
T=\operatorname{diag}([M, \quad M, I]), \quad \Delta x_{m}=T^{-1} \Delta x, \\
A_{m}=T^{-1} A T, \quad B_{m}=T^{-1} B, \quad \text { and } C_{m}=C T .
\end{array}
$$

The mode of concern $\left(\lambda_{i}\right)$, which is chosen from the eigenvalues of $A_{21}$, corresponding to the $0.49 \mathrm{~Hz}$ mode. Finally, the system with the $i^{\text {th }}$ mode to be evaluated can be expressed as

$$
\begin{aligned}
{\left[\begin{array}{c}
\Delta \dot{\delta}_{m i} \\
\Delta \dot{\omega}_{m i}
\end{array}\right] } & =\left[\begin{array}{ll}
a_{i 11} & a_{i 12} \\
a_{i 21} & a_{i 22}
\end{array}\right]\left[\begin{array}{c}
\Delta \delta_{m i} \\
\Delta \omega_{m i}
\end{array}\right]+\left[\begin{array}{c}
0 \\
b_{i 2}
\end{array}\right] u \\
y & =\left[\begin{array}{ll}
c_{i 1} & c_{i 2}
\end{array}\right]\left[\begin{array}{l}
\Delta \delta_{m i} \\
\Delta \omega_{m i}
\end{array}\right]+D u .
\end{aligned}
$$

The perturbed generator is $G_{6}$. The time responses of the perturbed KTH-NORDIC32 linear system are shown in Fig. 6 - 7. Fig. 6 illustrates the responses of the voltage magnitude deviation after the disturbance at $G_{6}$ while Fig. 7 illustrates the voltage angle differences. Figures (a) and (b) in each figure refer to the responses before and after filtering through network modeshapes, respectively. Note that the $0.49 \mathrm{~Hz}$ mode is selected for this analysis.

As shown in Fig. 6, the most observable signal is the signal combination $V_{37}+V_{48}$, followed in descendent order by $V_{38}, V_{37}, V_{48}, V_{50}$, and $V_{23}$ with the least observability.

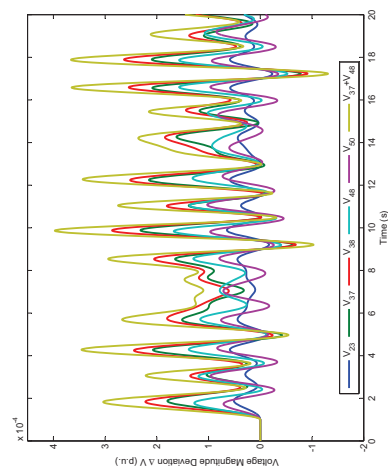

(a) Simulated responses

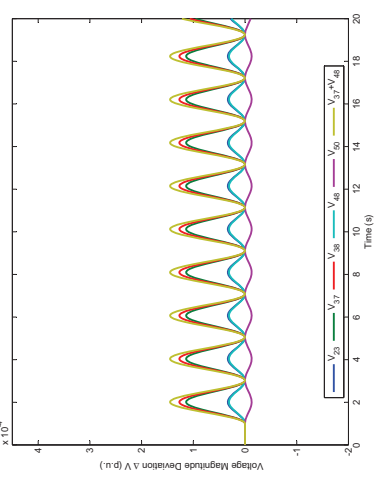

(b) After filtering through network modeshapes
Fig. 6. Voltage magnitude responses after a perturbation at $G_{6}$.

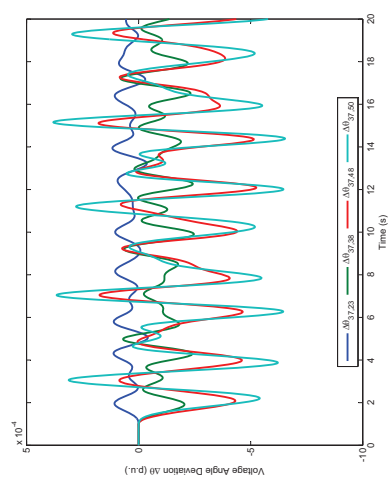

(a) Simulated responses

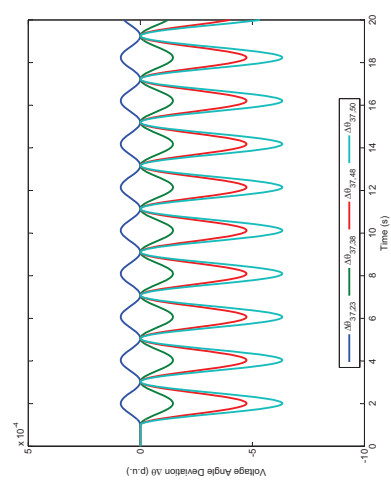

(b) After filtering through network modeshapes
Fig. 7. Voltage angle difference responses after a perturbation at $G_{6}$.

Overall, the magnitude of the signals are in accordance with the network modeshape of the nominal system as previously shown in Fig. 2 - 4. In other words, the voltage magnitude modeshapes indicate that the strongest signal is located at Bus 38, followed by Bus 37, Bus 48, and Bus 50 , respectively.

Comparing the responses before and after filtering through network modeshapes, more than one mode exist in the former while only one mode $(0.49 \mathrm{~Hz})$ is present in the latter. Observing Fig. 6(a), the mode appearance and magnitude can vary depending on fault locations. That is, the disturbance at $G_{6}$ considerably affects more than one mode causing a system response with several modal components. On the other hand, the responses in Fig. 6(b) are proportionally scaled; the unconcerned modes are removed, leaving only the mode of interest. Because of this network modeshape filter, not only is it possible to distinguish one mode from the rest, but also able to signify the distribution of the mode content among the signals.

For the voltage angle difference responses in Fig. 7, the largest element corresponds to $\Delta \theta_{37,50}$, descendingly followed by $\Delta \theta_{37,48}, \Delta \theta_{37,38}$, and $\Delta \theta_{37,23}$ with the smallest amplitude. This is in agreement with the voltage angle modeshape of the nominal system (Fig. 2 - 4) whereby the angle differences of the two edges (Bus 35 and Bus 50) yield the largest magnitude. Comparing among the figures, the use of network modeshape filtering helps not only scaling 
the contents of the mode onto the signals (the voltage angle difference), but also screening the undesirable modes out as illustrated in Fig. 7(a) - 7(b). The network modeshape filter is, therefore, precisely extracting the specific modal contributions at each bus angle, and their combination. This is the true inter-area mode content that should be expected from the resulting signals.

It has been suggested in [Korba et al. (2011)] to use bus voltage angle differences as input signals for damping purposes. However, this is only valid when the smaller angle is lower enough; in other words, the difference should be sufficiently large to be observable which is the case with the buses on the dominant path. When a signal from a non-dominant path is used, such as in the case with $\Delta \theta_{37,23}$, the amplitude of the response is much smaller than that of other signals from the dominant path. Hence, a proper choice of the bus angles used for this angle difference should consider the underlying inter-area mode distribution reflected by the network modeshapes in the dominant path. Otherwise, it is likely that the feedback signal will have a poor inter-area mode content that is not robust under different contingencies.

Although the signals $V_{38}, V_{23}$, and $\Delta \theta_{37,50}$ are among the most observable, they might not be practically available since it is less likely for the PMUs to be located at generator buses. The more prospective signals could be $V_{37}$, $V_{37}+V_{48}$, and/or $\Delta \theta_{37,48}$ because they are located at the high-voltage buses of the dominant path. This is consistent with the current field practice for deployment of PMUs [Chow et al. (2011)]. Depending on the availability of PMU measurements, different signal combinations, which will be discussed in a future publication, can be made available. Using these proposed signals as the inputs to PSS, it is further hypothesized that effective damping will be achieved.

\section{CONCLUSION}

This article presented a study showing the degree of persistence of dominant inter-area paths in the KTHNORDIC32 test system. The results confirm one of the hypotheses: the main dominant path persists under various contingencies. Based on contingency studies, suitable network variables to be used as PSS's input signals are proposed. To justify this selection, a small perturbation is applied to the nominal system to evaluate the proposed feedback signals (from the dominant path) and their combinations, as well as a comparison with a non-dominant path signal. The simulations assure the robustness and observability of the selected measurements.

Observe that the signals of choice are mainly dependent on PMU locations. At present, PMUs are not yet available everywhere in power systems, and it would be irrational to assume so. On the contrary, if the only available PMU signals were to be used, this study could offer an insightful and effective solution to locate the existing sets of signals that contain the highest possible content of inter-area modes.

\section{REFERENCES}

Chompoobutrgool, Y., Li, W., and Vanfretti, L. (2012). Development and Implementation of a Nordic Grid Model for Power System Small-Signal and Transient Stability Studies in a Free and Open Source Software. IEEE PES General Meeting.
Chompoobutrgool, Y. and Vanfretti, L. (2011). Linear Analysis of the KTH-NORDIC32 System. Technical Report Smarts-Lab-2011-001, KTH Royal Institute of Technology.

Chow, J., Beard, L., Patel, M., Quinn, P., Silverstein, A., Sobajic, D., and Vanfretti, L. (2011). Guildelines for Siting Phasor Measurement Units. Technical report, North American SynchroPhasor Initiative.

Chow, J., Sanchez-Gasca, J., Ren, H., and Wang, S. (2000). Power System Damping Controller Design Using Multiple Input Signals. IEEE Control Systems Magazine, 82-90.

Chow, J.H. (1982). Time-scale modeling of dynamic networks with applications to power systems. SpringerVerlag.

Glavic, M. and Van Cutsem, T. (2009). Wide-Area Detection of Voltage Instability From Synchronized Phasor Measurements. Part I, II. IEEE Transactions on Power Systems, 24(3), $1408-1425$.

Hauer, J., Trudnowski, D., and DeSteese, J. (2007). A perspective on WAMS analysis tools for tracking of oscillatory dynamics. IEEE PES General Meeting.

Kamwa, I., Beland, J., Trudel, G., Grondin, R., Lafond, C., and McNabb, D. (2006). Wide-Area Monitoring and Control at Hydro-Québec: Past, Present and Future. IEEE PES General Meeting.

Kamwa, I., Heniche, A., Trudel, G., Dobrescu, M., Grondin, R., and Lefebvre, D. (2005). Assessing the Technical Value of FACTS-Based Wide-Area Damping Control Loops. IEEE PES General Meeting.

Korba, P., Knazkins, V., and Larsson, M. (2011). Power System Stabilizer with Synchronized Phasor Measurements. $17^{\text {th }}$ Power Systems Computation Conference.

Larsen, E., Sanchez-Gasca, J., and Chow, J. (1995). Concepts of Design of FACTS Controllers to Damp Power Swings. IEEE Transactions on Power Systems, 10(2), 948-956.

Ledwich, G. and Palmer, E. (2000). Modal estimates from normal operation of power systems. IEEE PES Winter Meeting, 1527-1531.

Phadke, A. and Thorp, J. (2008). Synchronized Phasor Measurements and Their Applications. Springer.

Rogers, G. (1996). Demystifying Power System Oscillations. IEEE Computer Applications in Power, 30-35.

Sattinger, W. (2011). Application of PMU measurements in Europe TSO approach and experience. In IEEE Trondheim PowerTech 2011, 1 -4.

Van Cutsem, T. (2010). Description, Modelling and Simulation Results of a Test System for Voltage Stability Analysis. Technical Report Version 1, IEEE Working Group on Test Systems for Voltage stability analysis.

Vanfretti, L. and Chow, J. (2010). Analysis of Power System Oscillations for Developing Synchrophasor Data Applications. IREP Symposium - Bulk Power System Dynamics and Control.

Vanfretti, L. (2009). Phasor Measurement-Based State Estimation of Electric Power Systems and Linearized Analysis of Power System Network Oscillations. Ph.D. thesis, Rensselaer Polytechnic Institute, Troy, NY, USA.

Wei, X. (2002). Network Sensitivities of TCSC and SVC for Power Dispatching and Damping Control Design. Master's thesis, Rensselaer Polytechnic Institute. 